\title{
CONSTRUCTING COMPLETE CHAOTIC MAPS WITH RECIPROCAL STRUCTURES
}

WEIHONG HUANG

Received 26 October 2004

A class of unimodal complete chaotic maps $f=\left\{f_{L}, f_{R}\right\}$ with reciprocal functional forms $\left(f_{R}=1 / f_{L}\right)$ are examined. The necessary and sufficient conditions are provided to construct such maps. The theoretical conclusions are then verified by numerical simulations.

\section{Introduction}

The problem of determining nonlinear maps defined on an interval that preserves a prescribed invariant density is referred to as the inverse Frobenius-Perron problem (IFPP). The IFPP is traditionally treated through "conjugation approach," that is, variable transformation approach [11]. Other developments include the matrix approach [3,4], differential equation approach [10], the transverse to conjugation approach [6], and auxiliary variable approach [12]. The attempts have also been made in exploring some generic characteristics exhibited in some classes of chaotic maps that share similar analytical properties $[7,8,9]$. The current study intends to examine a class of complete chaotic maps with reciprocal structures.

To explain the terminology, we start with the well-known tent map defined by

$$
T_{2}(x)= \begin{cases}T_{L}(x)=2 x, & 0 \leq x<\frac{1}{2}, \\ T_{R}(x)=2(1-x), & \frac{1}{2} \leq x \leq 1,\end{cases}
$$

which is shown to preserve a uniform invariant density $\varphi_{T}(x) \equiv 1$, or, equivalently, a linear absolutely continuous invariant measure $\mu_{T}(x)=x$.

Performing a variable transformation $x=h_{f}(y) \doteq 2 y /(1+y)$ to $(1.1)$, we obtain a new map

$$
f(y)= \begin{cases}f_{L}(y)=\frac{2 y}{1-y}, & 0 \leq y<\frac{1}{3}, \\ f_{R}(y)=\frac{1-y}{2 y}, & \frac{1}{3} \leq y \leq 1 .\end{cases}
$$


The map $f$ defined by (1.2) is so called a topological conjugate of $T_{2}$. It preserves an invariant density $\varphi_{f}(y)=2 /(1+y)^{2}$. (All "invariant measure" mentioned in this paper is understood to be absolutely continuous with respect to Lebesgue measure.) The graphs of $f$ and $\varphi_{f}$ are illustrated in Figures 1.1(c) and 1.1(f), respectively.

If, instead, let $x=h_{g}(y)=4 / \pi \tan ^{-1}(y)$, we obtain from $T_{2}$ another conjugate:

$$
g(y)= \begin{cases}g_{L}(y)=\frac{2 y}{1-y^{2}}, & 0 \leq y<\sqrt{2}-1, \\ g_{R}(y)=\frac{1-y^{2}}{2 y}, & \sqrt{2}-1 \leq y \leq 1,\end{cases}
$$

which preserves an invariant density $\varphi_{g}(y)=4 / \pi\left(1+y^{2}\right)$. The graphs of $g$ and $\varphi_{g}$ are illustrated in Figures 1.2(b) and 1.2(f), respectively.

An interesting similarity is observed from $f$ and $g: f_{L}(y)=1 / f_{R}(y)$ and $g_{L}(y)=1 / g_{R}(y)$, for $y \in[0,1]$, that is, the left and the right branches are reciprocal to each other in their functional forms.

It is important to know whether $f$ and $g$ are just two incidents that happen to appear with such "reciprocal structure" in their functional forms or there exist a whole class of such maps. Since the class of two-to-one maps that are conjugated to the tent map forms only a subclass of two-to-one complete chaotic maps [12], we are enticed to explore the necessary and sufficient conditions for constructing general chaotic maps with reciprocal structures. We start with basic definitions in the next section.

\section{Unimodal complete chaotic maps}

We start with a general description of complete chaotic maps $[2,8]$ defined on the unitinterval: $I=[0,1]$. The particular choice of the unit-interval as a map's domain is not restrictive since any chaotic map defined on a closed interval can always be transformed to a map defined on the unit-interval through variable substitution (linear topological conjugation).

A map $f: I \rightarrow I$ is said to be unimodal if there exists a turning point $\hat{x} \in I$ such that the map $f$ can be expressed as

$$
f(x)=\min \left\{f_{L}(x), f_{R}(x)\right\}= \begin{cases}f_{L}(x), & 0 \leq x \leq \hat{x} \\ f_{R}(x), & \hat{x} \leq x \leq 1\end{cases}
$$

where $f_{L}:[0, \hat{x}] \rightarrow[0,1]$ and $f_{R}:[\hat{x}, 1] \rightarrow[0,1]$ are continuous, differentiable except possibly at finite points, monotonically increasing and decreasing, respectively, and onto the unit-interval in the sense that $f_{L}(0)=f_{R}(1)=0$ and $f_{L}(\hat{x})=f_{R}(\hat{x})=1$. Here, the "unimodal" is defined in the strict sense as introduced in [6], where the maximum of two-toone maps is restricted to unity so as to ensure complete chaos. This definition should not be confused with the generally accepted meaning of a "unimodal map" in the literature, where the maximum is immaterial. A unimodal map may not be chaotic.

For a unimodal complete chaotic map, given any $x \in I$, there exist two preimages of $f$, which are denoted as $x_{L}=f_{L}^{-1}(x)$ and $x_{R}=f_{R}^{-1}(x)$, respectively. The relationship between these two preimages can be captured by a branching function. 


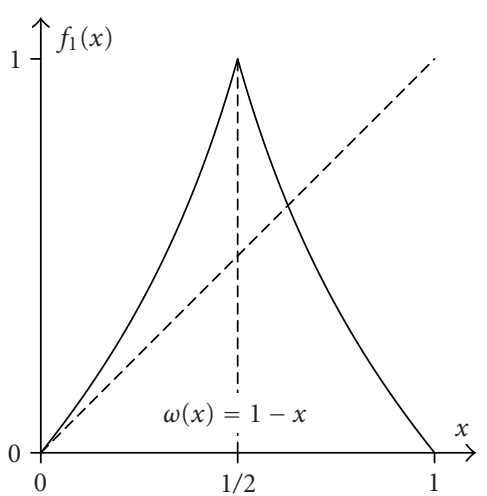

(a) $f_{L}(x)=\frac{x(5-x)}{(x+4)(1-x)}$.

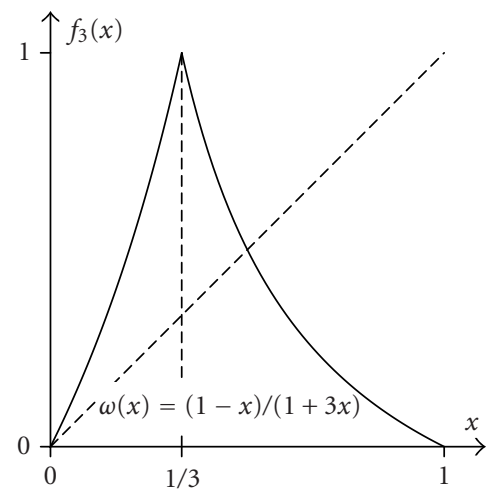

(c) $f_{L}(x)=\frac{2 x}{1-x}$.

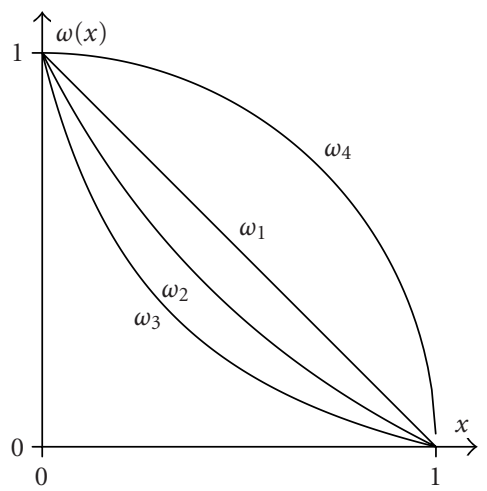

(e) $\omega(x)$.

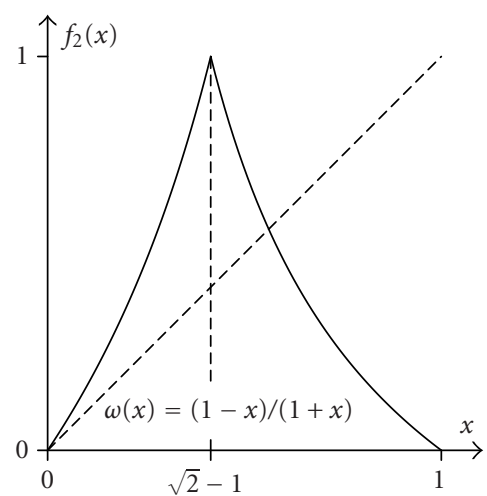

(b) $f_{L}(x)=\frac{x(3+x)}{2-x-x^{2}}$.

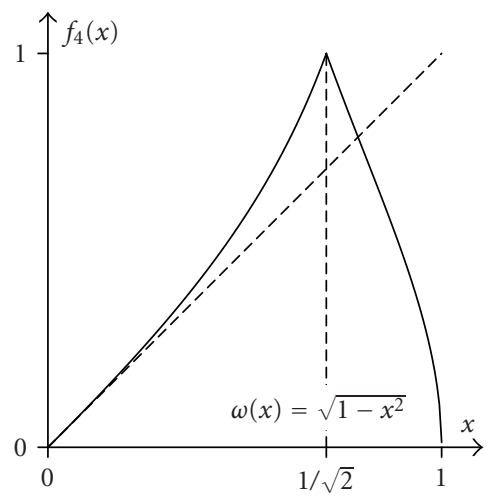

(d) $f_{L}(x)=\frac{(x-1) \sqrt{1-x^{2}}+3 x+1}{(x-1) \sqrt{1-x^{2}}-x+1}$.

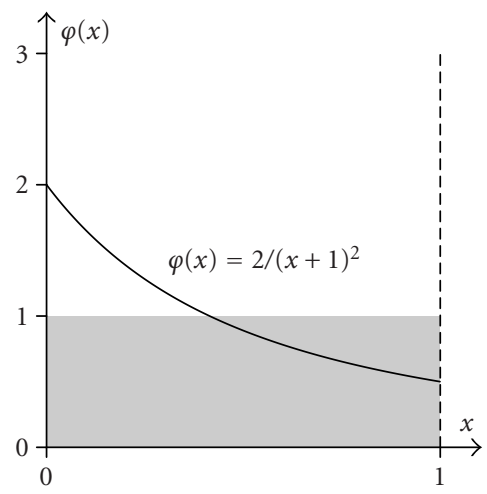

(f) Density $\varphi(x)$.

Figure 1.1. $f_{L}(x)=((x-1) \omega(x)+3 x+1) /((x+3) \omega(x)-x+1), x \in[0, \hat{x})$. 


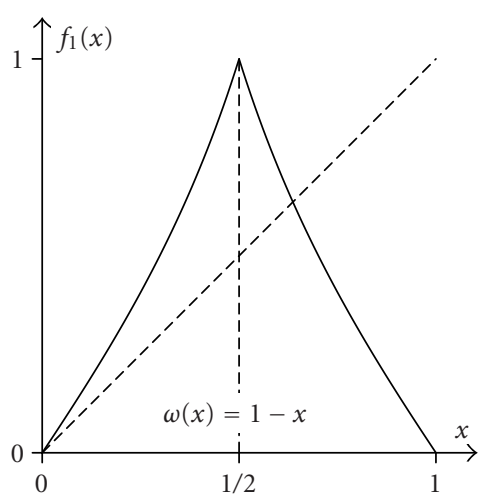

(a) $f_{L}(x)=\frac{x(3-x)}{2-x-x^{2}}$.

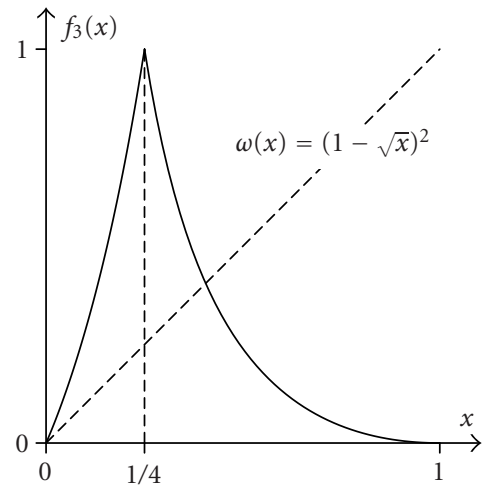

(c) $f_{L}(x)=\frac{(2-\sqrt{x}+x)^{2}}{x(\sqrt{x}-1)^{2}}$.

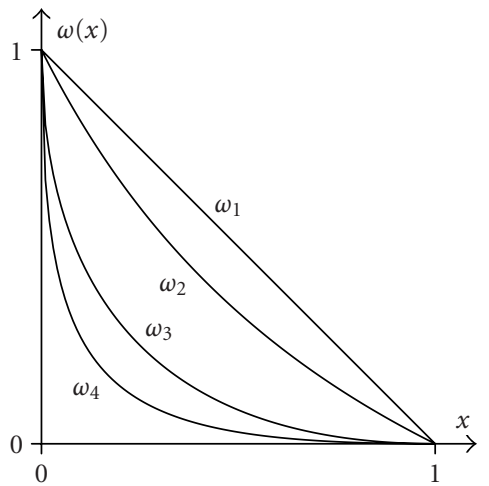

(e) $\omega(x)$.

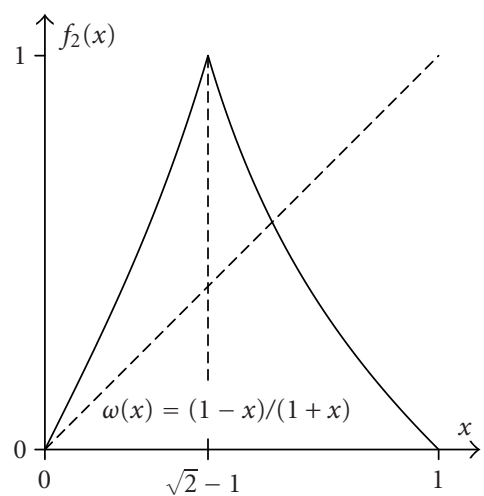

(b) $f_{L}(x)=\frac{2 x}{1-x^{2}}$.

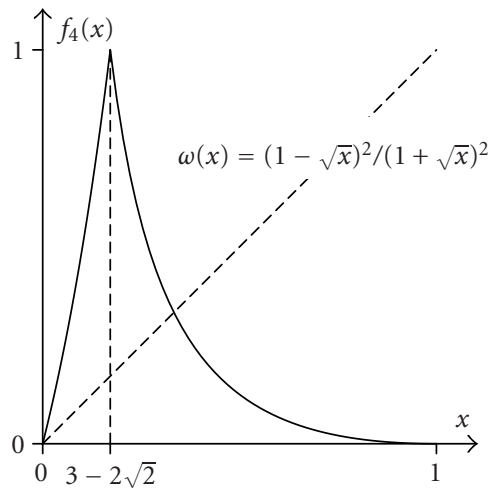

(d) $f_{L}(x)=\frac{4 x}{(1-x)^{2}}$.

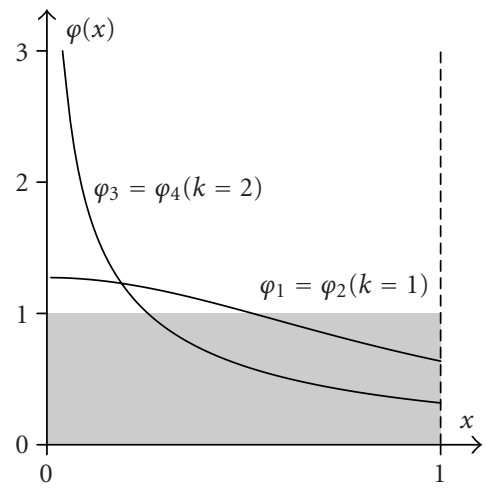

(f) $\varphi(x)=\frac{4}{k \pi} \frac{x^{1 / k}}{x\left(1+x^{2 / k}\right)}$.

Figure 1.2. $f_{L}(x)=((1+\sqrt[k]{x \omega(x)}+\sqrt[k]{x}-\sqrt[k]{\omega(x)}) /(1+\sqrt[k]{x \omega(x)}-\sqrt[k]{x}+\sqrt[k]{\omega(x)}))^{k}, x \in[0, \hat{x})$. 
Definition 2.1 (branching function). For a unimodal map $f$ defined by (2.1), a branching function $\bar{\omega}: I \rightarrow I$ is a one-to-one piecewise decreasing function that maps any point in one segment to a unique point in the other so that these two points have identical functional value. Mathematically, we have

$$
\begin{aligned}
\bar{\omega}(x) & =\left\{\omega(x), \omega^{-1}(x)\right\} \\
& = \begin{cases}\omega(x)=f_{R}^{-1}\left(f_{L}(x)\right), & x \in[0, \hat{x}), \\
\omega^{-1}(x)=f_{L}^{-1}\left(f_{R}(x)\right), & x \in[\hat{x}, 1] .\end{cases}
\end{aligned}
$$

Apparently, as a piecewise-defined function, $\bar{\omega}$ is differentiable except at a finite number of points. It is easy to see that

$$
\begin{aligned}
& \omega^{\prime}(x)<0, \quad \omega(0)=1, \quad \omega(\hat{x})=\hat{x}, \\
& \omega^{-1}(x)<0, \quad \omega(1)=0, \quad \omega^{-1}(\hat{x})=\hat{x} \text {. }
\end{aligned}
$$

Definition 2.2 (complete chaotic map). A unimodal map $f: I \rightarrow I$ is said to be complete chaotic if it is chaotic in a probabilistic sense that it preserves an absolutely continuous invariant measure. That is, for any set of $A \subset I$, there exists such an absolutely continuous invariant measure, denoted as $\eta$, that the following identity (Frobenius-Perron equation) is held:

$$
\eta(A)=\eta\left(f^{-1}(A)\right)
$$

Definition 2.3 (interval measure function). A function $h: I \rightarrow I$ is referred to as an interval measure function if $h$ meets the following requirements:

(i) $h(0)=0$ and $h(1)=1$;

(ii) $h$ is continuous;

(iii) $h^{\prime}(x)>0$ for almost all $x \in[0,1]$.

We will denote the set of interval measure functions as $\mathscr{H}$ and the set of interval measure functions that are analytic in $I$ as $\overline{\mathscr{H}}$. (A function is said to be analytic if it can be written in "closed form" in terms of known functions, constants, etc. so that it is infinitely differentiable. In this paper, however, the term of "analytic" is adopted mainly for its usage as an antonym of "piecewise-defined".)

Then for any invariant measure $\eta$, there exists a unique invariant measure function $\mu$ so that for any subinterval $A=[a, b] \subset I$, we have $\eta(A)=\mu(b)-\mu(a)$. Therefore, the Frobenius-Perron equation (2.5) can be rewritten as

$$
\mu(x)=\mu\left(f_{L}^{-1}(x)\right)+1-\mu\left(f_{R}^{-1}(x)\right), \quad \forall x \in[0,1],
$$

which leads to

$$
f(x)=\mu^{-1}(\mu(x)+1-\mu(\bar{\omega}(x))), \quad \forall x \in[0,1],
$$


or, equivalently,

$$
\begin{aligned}
& f_{L}(x)=\mu^{-1}(1+\mu(x)-\mu(\omega(x))), \quad \text { for } x \in[0, \hat{x}], \\
& f_{R}(x)=\mu^{-1}\left(1+\mu\left(\omega^{-1}(x)\right)-\mu(x)\right), \quad \text { for } x \in[\hat{x}, 1] .
\end{aligned}
$$

As long as $\mu$ and $\bar{\omega}(x)$ (or $\omega(x)$ ) as specified, $f_{L}(x)$ and $f_{R}(x)$ are uniquely determined. (Alternatively, we will refer $\mu$ as the invariant measure of $f$. The structure of (2.7) appears in [12] but its root can be traced back to [2].)

\section{Complete chaotic maps with reciprocal structures}

Now we are ready to examine the special class of complete chaotic maps that their two branches are reciprocal to each other, that is,

$$
f(x)= \begin{cases}f_{L}(x)=v(x), & 0 \leq x \leq \hat{x}, \\ f_{R}(x)=1 / v(x), & \hat{x} \leq x \leq 1\end{cases}
$$

For a general complete chaotic map, the domains of $f_{L}$ and $f_{R}$ are restricted to $[0, \hat{x}]$ and $[\hat{x}, 1]$, respectively. However, a reciprocal structure defined in (3.1) implies that the identity

$$
f_{R}(x)=1 / f_{L}(x)
$$

holds for all $x \in I$, which suggests that $f_{L}$, as an algebraic function, must be analytic in $I$, so is $f_{R}$.

To derive a set of sufficient conditions for the reciprocal structure, we notice first that (2.7) and (3.2) together yield

$$
\begin{gathered}
v(x)=\mu^{-1}(1+\mu(x)-\mu(\omega(x))), \quad x \in[0, \hat{x}), \\
1 / v(x)=\mu^{-1}\left(1+\mu\left(\omega^{-1}(x)\right)-\mu(x)\right), \quad x \in[\hat{x}, 1] .
\end{gathered}
$$

If $\omega$ is analytic in $I$ and diagonal-symmetric function in the sense of

$$
\omega(x)=\omega^{-1}(x), \quad x \in I,
$$

then the branching function $\bar{\omega}$ defined in (2.2) is not a piecewise-defined function anymore. In other words, $\bar{\omega}$ must be analytic over the whole unit interval $I$. If we further impose the requirement that $\mu$ (and hence $\mu^{-1}$ ) is analytic in $I$, then (3.3) and (3.4) imply that

$$
\mu^{-1}(1+\mu(x)-\mu(\omega(x))) \mu^{-1}(1+\mu(\omega(x))-\mu(x))=1 .
$$

Notice that, for any $x \in I$, the inequality of $1+\mu(x)-\mu(\omega(x)) \lessgtr 1$ suggests $1-\mu(x)+$ $\mu(\omega(x)) \gtrless 1$. To meet the condition (3.6), $\mu^{-1}$ should be analytic on $\bar{I}=[0,2]$ and should meet the following reciprocal condition:

$$
\mu^{-1}(1+x) \mu^{-1}(1-x)=1, \quad \forall x \in I .
$$


The above discussion leads to the following set of sufficient conditions for the construction of a unimodal complete chaotic map with a reciprocal structure.

Theorem 3.1. A unimodal complete chaotic map constructed through

$$
v(x)=\mu^{-1}(1-\mu(\omega(x))+\mu(x)),
$$

where

(i) $\omega$ is analytic in I and diagonal-symmetric in the sense of (3.5); and

(ii) $\mu$ is analytic in $[0,2]$ and its inverse satisfies identity (3.7), has a reciprocal structure given by (3.1) and will preserve the invariant measure $\mu$.

Before discussing in detail the sufficient conditions (3.5) and (3.7), we offer two examples.

Example 3.2. Let $\mu(x)=2 x /(x+1)\left(\mu^{-1}(x)=x /(2-x)\right)$. It can be verified that $\mu$ satisfies condition (3.7).

For any $\omega(x)$ that satisfies the condition $\omega^{-1}(x)=\omega(x)$, the induced transformation is given by

$$
f(x)= \begin{cases}\frac{(x-1) \omega(x)+3 x+1}{(x+3) \omega(x)-x+1}, & x \in[0, \hat{x}] \\ \frac{(x+3) \omega(x)-x+1}{(x-1) \omega(x)+3 x+1}, & x \in[\hat{x}, 1]\end{cases}
$$

Taking $\omega(x)=1-x,(3.9)$ simplifies to

$$
f_{1}(x)= \begin{cases}\frac{x(x-5)}{(x+4)(x-1)}, & x \in[0,1 / 2] \\ \frac{(x+4)(x-1)}{x(x-5)}, & x \in[1 / 2,1] .\end{cases}
$$

Taking $\omega(x)=(1-x) /(1+x),(3.9)$ simplifies to

$$
f_{2}(x)= \begin{cases}\frac{x(3+x)}{2-x-x^{2}}, & x \in[0, \sqrt{2}-1] \\ \frac{2-x-x^{2}}{x(3+x)}, & x \in[\sqrt{2}-1,1] .\end{cases}
$$

Taking $\omega(x)=(1-x) /(3 x+1),(3.9)$ simplifies to

$$
f_{3}(x)= \begin{cases}\frac{2 x}{1-x}, & x \in[0,1 / 3] \\ \frac{1-x}{2 x}, & x \in[1 / 3,1]\end{cases}
$$


Taking $\omega(x)=\sqrt{1-x^{2}},(3.9)$ simplifies to

$$
f_{4}(x)= \begin{cases}\frac{(x-1) \sqrt{1-x^{2}}+3 x+1}{(x+3) \sqrt{1-x^{2}}-x+1}, & x \in[0,1 / \sqrt{2}], \\ \frac{(x+3) \sqrt{1-x^{2}}-x+1}{(x-1) \sqrt{1-x^{2}}+3 x+1}, & x \in[1 / \sqrt{2}, 1] .\end{cases}
$$

The invariant density associated with (3.9) is always

$$
\varphi(x)=\frac{2}{(x+1)^{2}} .
$$

Figures 1.1(a), 1.1(b), 1.1(c), and 1.1(d) illustrate the functional graphs of $f_{i}, i=1$, $2, \ldots, 4$. Corresponding branching functions are shown in Figure 1.1(e). Figure 1.1(f) depicts the relevant invariant densities.

Example 3.3. For $\mu(x)=4 / \pi \tan ^{-1}\left(x^{1 / k}\right)$, it is easy to verify that $\mu^{-1}(1+x) \mu^{-1}(1-x)=1$. Since $\mu^{-1}(x)=\tan ^{k}((\pi / 4) x)$, the left branch is thus given by

$$
\begin{aligned}
f_{L}(x) & =\tan ^{k}\left(\frac{\pi}{4}(1+\mu(x)-\mu(\omega(x)))\right) \\
& =\tan ^{k}\left(\pi / 4+\tan ^{-1}\left(x^{1 / k}\right)-\tan ^{-1}(\omega(x))^{1 / k}\right) .
\end{aligned}
$$

Using the formula

$$
\tan \left(\frac{\pi}{4}(1+x)\right)=\frac{1+\tan (\pi / 4) x}{1-\tan (\pi / 4) x}
$$

we have

$$
f_{L}(x)=\left(\frac{1+\sqrt[k]{x \omega(x)}+\sqrt[k]{x}-\sqrt[k]{\omega(x)}}{1+\sqrt[k]{x \omega(x)}-\sqrt[k]{x}+\sqrt[k]{\omega(x)}}\right)^{k}
$$

and $f_{R}=1 / f_{L}$.

Taking $k=1$ and $\omega(x)=1-x,(3.17)$ simplifies to

$$
f_{1}(x)= \begin{cases}\frac{x(3-x)}{2-x-x^{2}}, & x \in[0,1 / 2] \\ \frac{2-x-x^{2}}{x(3-x)}, & x \in[1 / 2,1]\end{cases}
$$

Taking $k=1$ and $\omega(x)=(1-x) /(1+x),(3.17)$ simplifies to

$$
f_{2}(x)= \begin{cases}\frac{2 x}{1-x^{2}}, & x \in[0, \sqrt{2}-1] \\ \frac{1-x^{2}}{2 x}, & x \in[\sqrt{2}-1,1] .\end{cases}
$$


Taking $k=2$ and $\omega(x)=(1-\sqrt{x})^{2},(3.17)$ simplifies to

$$
f_{3}(x)= \begin{cases}\frac{(2-\sqrt{x}+x)^{2}}{x(\sqrt{x}-1)^{2}}, & x \in[0,1 / \sqrt{2}], \\ \frac{x(\sqrt{x}-1)^{2}}{(2-\sqrt{x}+x)^{2}} & x \in[1 / \sqrt{2}, 1] .\end{cases}
$$

Taking $k=2$ and $\omega(x)=(1-\sqrt{x})^{2} /(1+\sqrt{x})^{2},(3.17)$ simplifies to

$$
f_{4}(x)= \begin{cases}\frac{4 x}{(1-x)^{2}}, & x \in[0,3-2 \sqrt{2}], \\ \frac{(1-x)^{2}}{4 x}, & x \in[3-2 \sqrt{2}, 1] .\end{cases}
$$

The invariant density associated with (3.17) is

$$
\varphi(x)=\frac{4}{k \pi} \frac{x^{1 / k}}{x\left(1+x^{2 / k}\right)} .
$$

Figures 1.2(a), 1.2(b), 1.2(c), and 1.2(d) illustrate the functional graphs of $f_{i}, i=1$, $2, \ldots, 4$. Corresponding branching functions are shown in Figure 1.2(e). Figure 1.2(f) depicts the relevant invariant densities.

\section{More on the sufficient conditions}

It needs to be emphasized that, although conditions (3.5) and (3.7) together are not the necessary conditions for a unimodal complete chaotic map to have a reciprocal structure, each individual condition is indeed a necessary condition if the other is assumed to hold true.

Substituting $\omega=\omega^{-1}$ into (3.3) and (3.4), we obtain

$$
\mu(v(x))+\mu(1 / v(x))=2, \quad \forall x \in[0,1]
$$

which is equivalent to

$$
\mu(x)+\mu(1 / x)=2, \quad \forall x \in[0,1] .
$$

Condition (4.2) thus requires $\mu$ be analytic on whole real line $\mathbb{R}$ and the invariant density satisfies the following property

$$
\varphi(1 / x)=x^{2} \varphi(x)
$$

Basing on the above reasonings, we should be able to infer that condition (3.7) and (4.2) imply each other.

Let $\overline{\mathfrak{N}}$ be the set of interval measure functions which is analytic in $\mathbb{R}$ and fulfill the condition (4.2) (or, equivalently, (3.7)). The following lemma sheds light on the direction of constructing such invariant measures. 
Lemma 4.1. (i) Average rule for $\overline{\mathfrak{N}}$ : if $\mu_{i} \in \overline{\mathfrak{N}}, i=1,2$, for any $\alpha \in[0,1]$, let $\mu \doteq \alpha \mu_{1}+(1-$ $\alpha) \mu_{2}$, then $\mu \in \mathfrak{N}$.

(ii) Product rule for $\overline{\mathfrak{N}}$ : if $\mu_{i} \in \overline{\mathfrak{N}}$ and $k_{i}>0, i=1,2$, let $\mu^{-1} \doteq\left(\mu_{1}^{-1}\right)^{\alpha}\left(\mu_{2}^{-1}\right)^{\beta}$, then $\mu \in \mathfrak{N}$.

(iii) Composition rule for $\overline{\mathfrak{N}}$ : let $h: I \rightarrow I$ be an analytic interval measure function satisfies the condition

$$
h(1 / x)=1 / h(x), \quad \forall x \in I
$$

then for any $\mu \in \overline{\mathfrak{N}}, \tilde{\mu} \doteq \mu \circ h \in \mathfrak{N}$.

Proof. Part (i) follows from (3.17).

Part (ii) follows from (3.7).

We only need to prove Part (iii). If $h(1 / x)=1 / h(x)$, then

$$
\mu(h(x))+\mu(h(1 / x))=\mu(h(x))+\mu(1 / h(x))=2 .
$$

Remark 4.2. The implication of the product rule is, for any $\mu \in \overline{\mathfrak{N}}$ and $k>0$, we have

$$
\mu_{k}(x)=\mu\left(x^{k}\right) \in \overline{\mathfrak{N}} \text {. }
$$

Together with the average rule, we may say that, if $\mu_{i} \in \overline{\mathfrak{N}}$ and $k_{i}>0$, then for any $\alpha \in$ $[0,1]$, we have

$$
\mu_{\alpha}(x) \doteq \alpha \mu_{1}\left(x^{k_{1}}\right)+(1-\alpha) \mu_{2}\left(x^{k_{2}}\right) \in \overline{\mathfrak{N}}
$$

Let $\bar{\Omega}$ be the set of analytic diagonal-symmetric functions. The simplest analytic function that satisfies the diagonal-symmetry condition is related to the symmetric branching function, that is, $\omega(x)=1-x$.

Other members of $\bar{\Omega}$ include

$$
\begin{aligned}
& \omega_{1}(x)=\left(\frac{1-x^{k}}{1+x^{k}}\right)^{1 / k}, \quad k>0, \\
& \omega_{2}(x)=\left(1-x^{k}\right)^{1 / k}, \quad k>0, \\
& \omega_{3}(x)=1-\left(1-(1-x)^{k}\right)^{1 / k}, \quad k>0, \\
& \omega_{4}(x)=\frac{\beta^{2}(1-x)}{(1-\beta)^{2} x+\beta^{2}(1-x)}, \quad 0<\beta<1, \\
& \omega_{5}(x)=\frac{2}{\pi} \tan ^{-1}\left(\frac{1}{\tan (\pi / 2 x)}\right) .
\end{aligned}
$$

The proof of the following lemma is straightforward.

Lemma 4.3. The composition rule for $\bar{\Omega}:$ for any $h \in \overline{\mathcal{H}}$ and any $\omega \in \bar{\Omega}$

$$
\tilde{\omega}(x)=h^{-1}(\omega(h(x))) \in \bar{\Omega} .
$$

Furthermore, if $\omega$ branches at $\hat{x}$, that is, $\omega(\hat{x})=\hat{x}$, then $\tilde{\omega}$ branches at $h^{-1}(\hat{x})$. 
The abundance of analytic diagonal-symmetric functions is thus manifested by the following constructive properties of $h$.

Properties of analytic interval measure functions:

(1) If $h \in \overline{\mathscr{H}}$, then $h^{-1} \in \overline{\mathscr{H}}$;

(2) If $h_{i} \in \overline{\mathscr{H}}, i=1,2$, then their compositions defined by $h_{12}=h_{1}\left(h_{2}(x)\right)$ and $h_{21}=$ $h_{2}\left(h_{1}(x)\right)$ also belong to $\overline{\mathscr{H}}$;

(3) If $h_{i} \in \overline{\mathscr{H}}, i=1,2$, then their average function given by $\epsilon h_{1}+(1-\epsilon) h_{2}$, with $0 \leq$ $\epsilon \leq 1$, also belongs to $\overline{\mathscr{H}}$;

(4) If $h_{i} \in \overline{\mathscr{H}}, i=1,2$, then their product given by $h_{1}^{k} h_{2}^{l}$, with $k, l>0$ for any real numbers $k$ and $l$, also belongs to $\overline{\mathcal{H}}$.

One direct implication of the above properties is, for any $h \in \overline{\mathscr{H}}$, given a $\beta>0, h\left(x^{\beta}\right)$ and $h^{\beta}(x)$ are analytic interval measure functions as well.

With the composition rules for $\overline{\mathfrak{A}}$ and $\bar{\Omega}$, we are able to arrive at the following important conclusion.

THEOREM 4.4. For any unimodal complete chaotic map $f$ that has a reciprocal structure, its conjugate defined by $\tilde{f}_{h}(x)=h^{-1}(f(h(x)))$, preserves the reciprocal structure if and only if $h: I \rightarrow I$ is an analytic interval measure function that satisfies condition (4.4).

Example 4.5. Let $\mu_{1}(x)=2 x /(x+1)$. Since $\mu_{1} \in \mathfrak{N}$, then for any given interval measure function $h$ satisfying the condition (4.4), the invariant measure given by

$$
\mu(x)=\mu_{1}(h(x))=\frac{2 h(x)}{h(x)+1}
$$

is also a member of $\mathfrak{N}$. The inverse of $\mu$ is given by

$$
\mu^{-1}(x)=h^{-1}\left(\frac{x}{2-x}\right)
$$

Therefore, for any $\omega$ satisfying the condition $\omega^{-1}=\omega$, a complete chaotic map with reciprocal structure can be constructed with

$$
\begin{aligned}
f_{L}(x) & =\mu^{-1}(1+\mu(x)-\mu(\omega(x))) \\
& =h^{-1}\left(\frac{h(x) h(\omega(x))+3 h(x)-h(\omega(x))+1}{h(x) h(\omega(x))+3 h(\omega(x))-h(x)+1}\right) .
\end{aligned}
$$

The invariant density is

$$
\varphi(x)=\mu_{1}^{\prime}(h(x)) h^{\prime}(x)=\frac{2 h^{\prime}(x)}{(1+h(x))^{2}} .
$$

An interesting instance appears when $f$ is symmetric, that is, $\omega(x)=1-x$, in which case

$$
\begin{gathered}
f_{L}(x)=h^{-1}\left(\frac{h(x) h(1-x)+3 h(x)-h(1-x)+1}{h(x) h(1-x)+3 h(1-x)-h(x)+1}\right), \\
f_{R}(x)=f_{L}(1-x)=1 / f_{L}(x) .
\end{gathered}
$$


Let $h(x)=x^{k}, k>0$, we have $h(1 / x)=1 / h(x)$, therefore,

$$
f_{L}(x)=\left(\frac{x^{k}(1-x)^{k}+3 x^{k}-(1-x)^{k}+1}{x^{k}(1-x)^{k}+3(1-x)^{k}-x^{k}+1}\right)^{1 / k} .
$$

It is easy to see that $f_{L}(1-x)=1 / f_{L}(x)$. Figure 4.1 illustrates map (4.16) with several typical $k$ values along with realized densities generated from some typical trajectories. (All realized densities in this research are simulated with Maple 8.0 with 50000 iterations, 100 intervals, and an initial value $x_{0}=\pi / 10$.) The invariant densities related are

$$
\varphi(x)=\frac{2 k x^{k-1}}{\left(1+x^{k}\right)^{2}} .
$$

A special class of complete chaotic maps with reciprocal structures are related to the conjugation class of the tent map defined in (1.1), or, more generally, a tilted-tent map defined by

$$
T_{\sigma}(x)= \begin{cases}T_{L}(x)=\sigma x, & 0 \leq x<1 / \sigma, \\ T_{R}(x)=\frac{\sigma(1-x)}{\sigma-1}, & 1 / \sigma \leq x \leq 1,\end{cases}
$$

where $\sigma>1$.

For a given invariant measure $\mu$, the class of complete chaotic unimodal maps that are conjugated to the titled-tent map consists of a subset of all complete chaotic unimodal complete chaotic maps that preserving the very invariant measure. For convenience of reference, we will call this subset as $C$-map. A general formulation of $C$-map is

$$
C(x)= \begin{cases}C_{L}(x)=\mu^{-1}\left(\frac{\mu(x)}{\mu(\hat{x})}\right), & 0 \leq x<\hat{x}, \\ C_{R}(x)=\mu^{-1}\left(\frac{1-\mu(x)}{1-\mu(\hat{x})}\right), & \hat{x} \leq x \leq 1,\end{cases}
$$

which is topologically conjugated in the metric sense to $T_{1 / \mu(\hat{x})}$.

In contrast to the general formulation of (2.7), where the specification of branching function is independent of the invariant measure preserved, the branching function for a $C$-map defined by (4.19) is related closely to the invariant measure (conjugating function). Comparing (4.19) with (2.7), we must have $\mu(x)-\mu(\omega(x))+1=\mu(x) / \mu(\hat{x})$, which suggests that

$$
\begin{aligned}
\omega(x) & =\mu^{-1}\left(1-\left(\frac{1}{\mu(\hat{x})}-1\right) \mu(x)\right), \\
\omega^{-1}(x) & =\mu^{-1}\left(\frac{\mu(\hat{x})}{1-\mu(\hat{x})}(1-\mu(x))\right) .
\end{aligned}
$$

Therefore, the diagonal-symmetric condition (3.5) requires

$$
\mu(\hat{x})=1 / 2,
$$



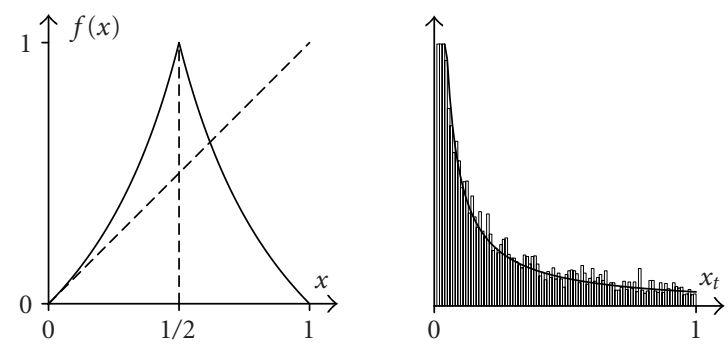

(a) $k=1 / 4$.
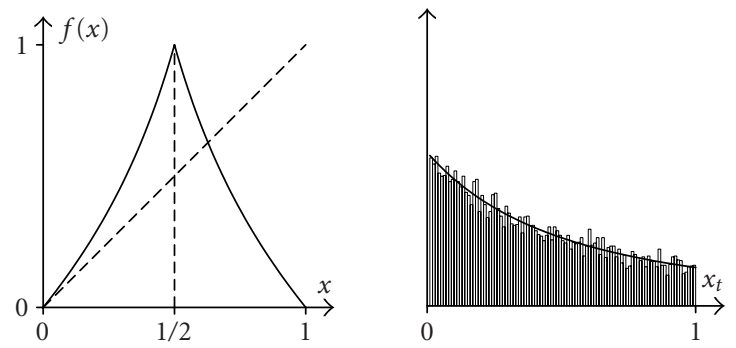

(b) $k=1$.
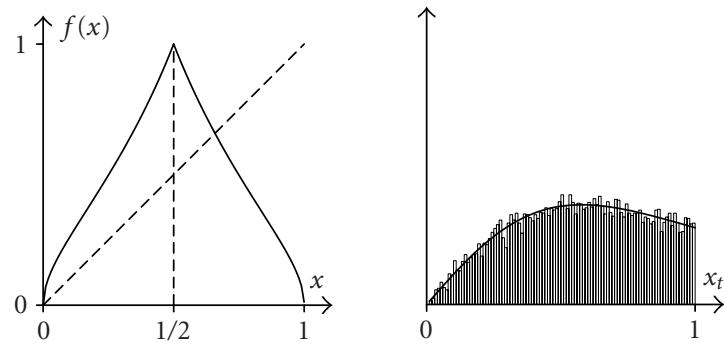

(c) $k=2$.
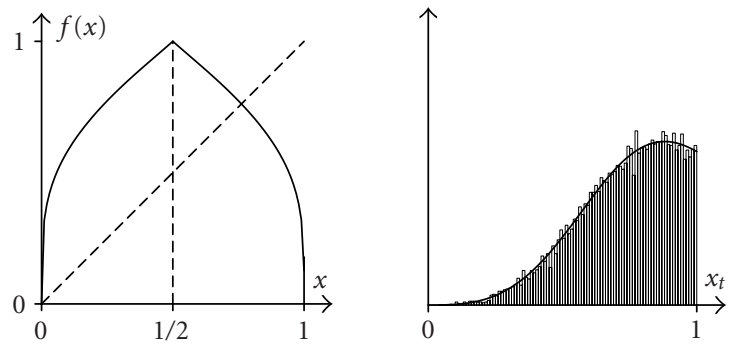

(d) $k=4$.

Figure 4.1. $f_{L}(x)=\left(\left(x^{k}(1-x)^{k}+3 x^{k}-(1-x)^{k}+1\right) /\left(x^{k}(1-x)^{k}+3(1-x)^{k}-x^{k}+1\right)\right)^{1 / k}, k>0$. 
that is, the $C$-map must be the one conjugating to the tent map $T_{2}$. As long as (4.21) is imposed, we have

$$
\omega(x)=\mu^{-1}(1-\mu(x))=\omega^{-1}(x),
$$

regardless of the definition of $\mu$. Under such circumstance, condition (3.7) alone becomes both a necessary condition and sufficient condition for a $C$-map to have reciprocal structure.

Corollary 4.6. A C-map defined by (4.19) has a reciprocal structure if and only if (4.21) and (3.7) hold true.

\section{Lyapunov exponents}

The simplicity and analytical characteristics of complete chaotic maps with the reciprocal structure simplify the calculations of various statistical properties of chaotic dynamics. Due to the limited scope, we will illustrate this point with the Lyapunov exponent (LC). The Lyapunov exponent of a chaotic process defined by

$$
\Lambda=\lim _{N \rightarrow \infty} \frac{1}{N} \sum_{t=0}^{N} \ln \left|f^{\prime}\left(x_{t}\right)\right|=\int_{0}^{1} \varphi(x) \ln \left|f^{\prime}(x)\right| d x
$$

characterizes the degree of chaosity through measuring the average divergence rate of nearby trajectories.

The simplicity in the functional form of complete chaotic maps with the reciprocal structure map provides us a great advantage in the evaluation $\Lambda$ both analytically and numerically. If $f$ is constructed through (3.8), it follows from

$$
\mu(f(x))=1+|\mu(x)-\mu(\omega(x))|, \quad \text { for } x \in[0,1],
$$

that

$$
\left|f^{\prime}(x)\right| \varphi(f(x))=\varphi(x)-\varphi(\omega(x)) \omega^{\prime}(x)
$$

Therefore,

$$
\begin{aligned}
\Lambda & =\int_{0}^{1} \varphi(x) \ln \frac{\varphi(x)-\varphi(\omega(x)) \omega^{\prime}(x)}{\varphi(f(x))} d x \\
& =\int_{0}^{1} \varphi(x) \ln \left(\varphi(x)-\varphi(\omega(x)) \omega^{\prime}(x)\right) d x-\int_{0}^{1} \varphi(x) \ln \varphi(f(x)) d x .
\end{aligned}
$$

Now that the ergodicity of the complete chaotic map $f$ implies that

$$
\int_{0}^{1} \varphi(x) \ln \varphi(f(x)) d x=\int_{0}^{1} \varphi(x) \ln \varphi(x) d x,
$$

we finally have

$$
\Lambda=\int_{0}^{1} \varphi(x) \ln \left(1-\frac{\varphi(\omega(x))}{\varphi(x)} \omega^{\prime}(x)\right) d x
$$


Formula (5.6) enables us to explore LC under different structures of $\omega$ and $\varphi$.

Numerical calculation with (5.6) is much simpler than that with (5.1). For instance, consider the case in which $f$ is symmetric, that is, $\omega(x)=1-x$, we have

$$
\Lambda=\int_{0}^{1} \varphi(x) \ln \left(1+\frac{\varphi(1-x)}{\varphi(x)}\right) d x
$$

Further consider two special cases:

(1) $\varphi$ is symmetric as well, that is, $\varphi(x)=\varphi(1-x)$, then we arrive at a maximum possible Lyapunov exponent $\Lambda=\int_{0}^{1} \ln 2 \varphi(x) d x=\ln 2$, a conclusion drawn in [5].

(2) $\varphi$ is odd-symmetric, that is, $\varphi(x)=1-\varphi(1-x)$, we have

$$
\Lambda=\int_{0}^{1} \varphi(x) \ln \left(1+\frac{1-\varphi(x)}{\varphi(x)}\right) d x=-\int_{0}^{1} \varphi(x) \ln (\varphi(x)) d x=\text { entropy of } \varphi
$$

Moreover, with (5.6) in hand, we are able to evaluate comparative statics with respect to the effects of various parameters on the Lyapunov component directly.

\section{Conclusion}

We have provided in theory a set of sufficient conditions so that a class of unimodal complete chaotic maps with reciprocal structure can be constructed explicitly with closed formulas. Examples and numerical simulations are provided, which have shown to be consistent with the theoretical findings. Our results greatly extend the applicability of the general formulation of unimodal complete chaotic maps given by (2.7).

\section{References}

[1] A. Boyarsky and P. Góra, Laws of Chaos. Invariant Measures and Dynamical Systems in One Dimension, Probability and Its Applications, Birkhäuser, Massachusetts, 1997.

[2] S. V. Ershov and G. G. Malinetskii, The solution of the inverse problem for the Perron-Frobenius equation, U.S.S.R. Comput. Math. and Math. Phys. 28 (1988), no. 5, 136-141.

[3] N. Friedman and A. Boyarsky, Construction of ergodic transformations, Adv. in Math. 45 (1982), no. 3, 213-254.

[4] P. Góra and A. Boyarsky, A matrix solution to the inverse Perron-Frobenius problem, Proc. Amer. Math. Soc. 118 (1993), no. 2, 409-414.

[5] S. Grossmann and S. Thomae, Invariant distributions and stationary correlation functions of one-dimensional discrete processes, Z. Naturforsch. 32a (1977), no. 12, 1353-1363.

[6] G. Gyögyi and P. Szépfalusy, Fully developed chaotic 1-d maps, Z. Phys. B 55 (1984), no. 2, 179-186.

[7] W. Huang, Characterizing chaotic processes that generate uniform invariant density, Chaos Solitons Fractals 25 (2005), no. 2, 449-460.

[8] Constructing an opposite map to a specified chaotic map, Nonlinearity 18 (2005), no. 3, 1375-1391.

[9] - On complete chaotic maps with tent-map-like structures, Chaos Solitons Fractals 24 (2005), no. 1, 287-299.

[10] S. Koga, The inverse problem of Frobenius-Perron equations in $1 D$ difference systems. $1 D$ map idealization, Progr. Theoret. Phys. 86 (1991), no. 5, 991-1002. 


\section{Complete chaotic maps with reciprocal structures}

[11] A. Lasota and M. C. Mackey, Probabilistic Properties of Deterministic Systems, Cambridge University Press, Cambridge, 1985.

[12] D. Pingel, P. Schmelcher, and F. K. Diakonos, Theory and examples of the inverse FrobeniusPerron problem for complete chaotic maps, Chaos 9 (1999), no. 2, 357-366.

Weihong Huang: Nanyang Technological University, Nanyang Avenue, Singapore 639798

E-mail address: awhhuang@ntu.edu.sg 


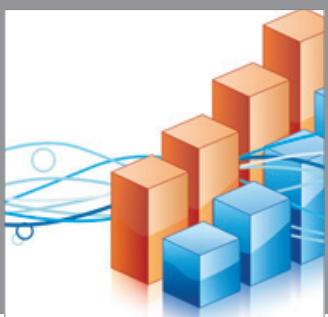

Advances in

Operations Research

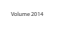

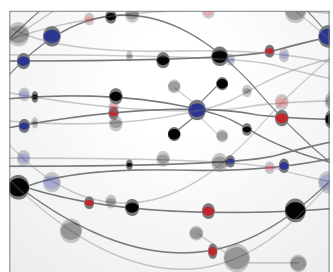

\section{The Scientific} World Journal
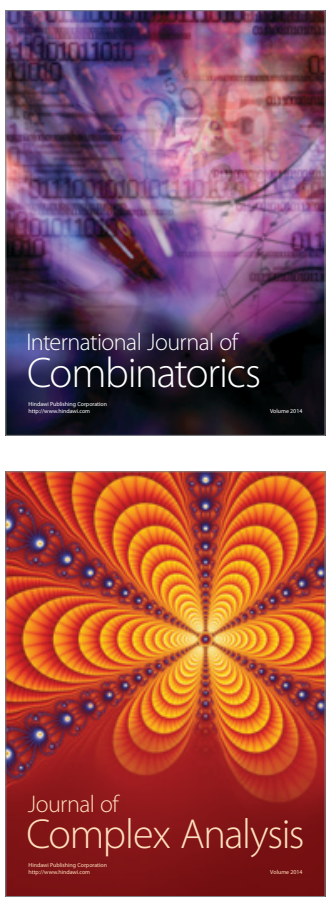

International Journal of

Mathematics and

Mathematical

Sciences
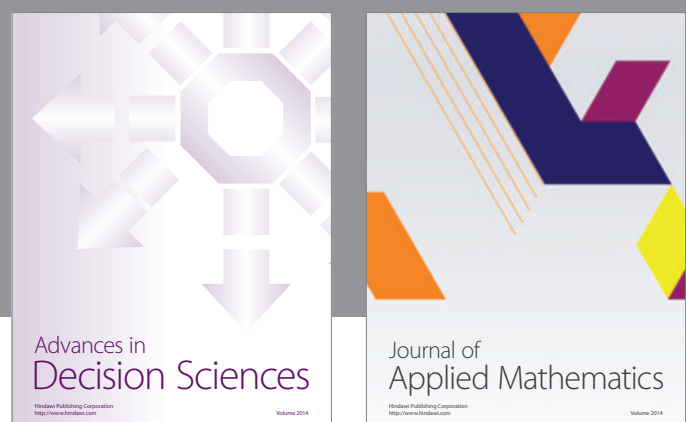

Journal of

Applied Mathematics
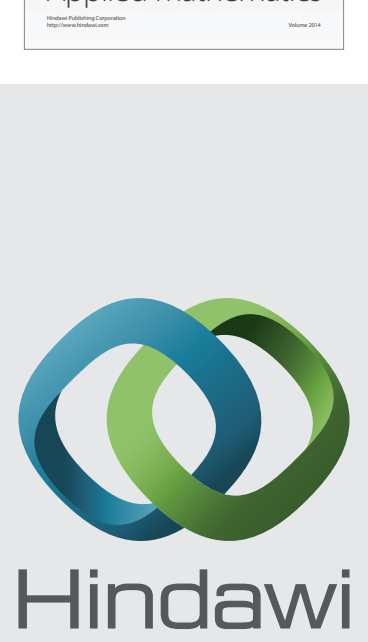

Submit your manuscripts at http://www.hindawi.com
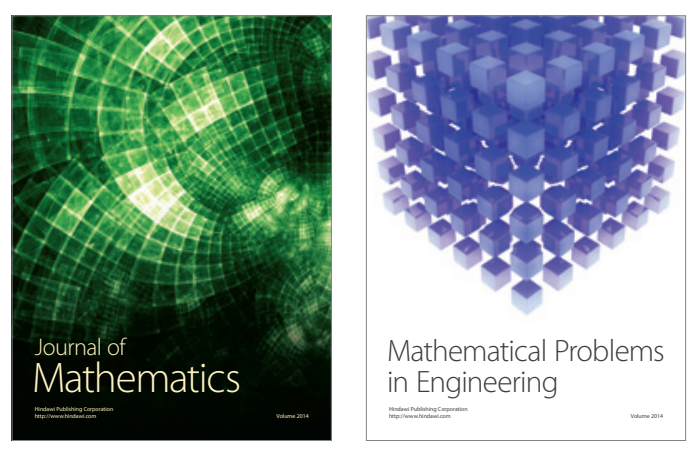

Mathematical Problems in Engineering
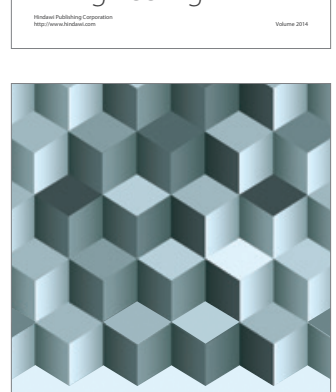

Journal of

Function Spaces
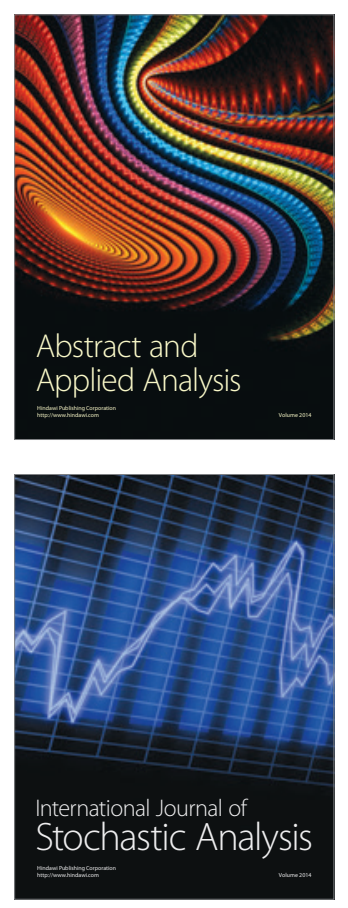

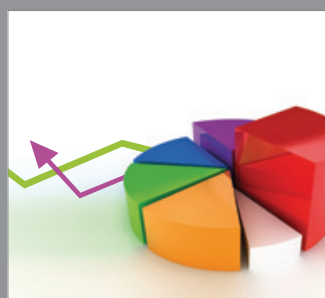

ournal of

Probability and Statistics

Promensencen
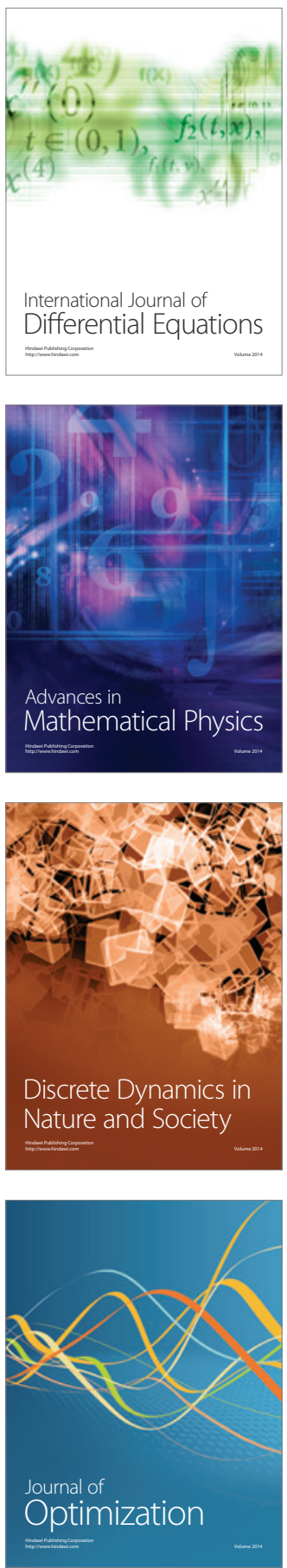